\title{
ASPIRACIONES Y PROYECTOS DE FUTURO DE JÓVENES ESTUDIANTES SECUNDARIOS EN CHILE: EL SOPORTE FAMILIAR Y SU INFLUENCIA EN LAS DECISIONES EDUCATIVO-LABORALES ${ }^{1}$
}

\author{
Leandro Sepúlveda V." \\ Universidad Alberto Hurtado (UAH), Santiago, Región - Metropolitana, Chile
}

RESUMEN: Este artículo analiza las aspiraciones y los proyectos educativos postsecundarios de jóvenes estudiantes chilenos, considerando el rol y la incidencia del soporte familiar en este proceso. Las aspiraciones de cursar estudios superiores es una realidad extendida entre los jóvenes, aunque es posible identificar racionalidades diversas asociadas a las opciones formativas y los itinerarios previstos para alcanzar estos objetivos. El entorno familiar juega un rol importante en la construcción del proyecto personal de los estudiantes, existiendo una alta valoración del soporte que estos brindan para el logro de las metas educacionales. Con todo, la experiencia varía en relación a las características de este apoyo para la toma de decisiones individuales, como también en las estrategias de presión o exigencias para el cumplimiento de obligaciones y objetivos en la educación superior, evidenciándose diferencias importantes de acuerdo al nivel socioeconómico de las familias.

Palabras claves: Aspiraciones. Transición Educación-Trabajo. Estudiantes Secundarios. Participación de Padres. Soporte Familiar.

\section{ASPIRAC̣̃̃ES E PROJETOS DE FUTURO DE JOVENS ESTUDANTES SECUNDARISTAS NO CHILE: O SUPORTE FAMILIAR E SUA INFLUÊNCIA NAS DECISÕES SOBRE EDUCAÇÃO E TRABALHO}

RESUMO: Este artigo analisa as aspirações e os projetos educativos pós-ensino médio de jovens estudantes chilenos, considerando o papel e a incidência do suporte familiar nesse processo. O desejo de cursar o ensino superior é uma realidade presente entre os jovens, ainda que seja possível identificar diversas lógicas associadas às opções formativas e aos itinerários previstos para alcançar esse objetivo. O entorno familiar tem um papel importante na construção do projeto pessoal dos estudantes, havendo uma grande valorização do apoio existente para se conquistar as metas educacionais. Contudo, a experiência varia em relação às características de tal apoio para a tomada de decisões individuais, como também em relação às estratégias

“Antropólogo Social, Doctor en el Estudio de las Sociedades Latinoamericanas de la Universidad ARCIS. Profesor e Investigador del Centro de Investigación y Desarrollo de la Educación, CIDE, de la Universidad Alberto Hurtado, Santiago de Chile. E-mail: < Isepulve@cide.cl>. 
de pressão ou exigências para o cumprimento de obrigações e objetivos na educação superior, evidenciando-se, assim, diferenças importantes de acordo com o nível socioeconômico das famílias.

Palavras-chave: Aspirações. Transição Educação-Trabalho. Estudantes Secundaristas. Participação dos Pais. Suporte Familiar.

\title{
PROJECTS AND FUTURE ASPIRATIONS OF YOUNG HIGH SCHOOL STUDENTS IN CHILE: SUPPORT FROM FAMILY AND ITS INFLUENCE ON THE EDUCATIONAL AND EMPLOYMENT DECISIONS
}

\begin{abstract}
This article analyzes the aspirations and post-secondary educational projects of Chilean students, considering the role and impact of family support in this process. The aspirations of continuing higher education is a widespread reality among young people, although it is possible to identify various rationalities associated with education options and itineraries foreseen to achieve these objectives. The family environment plays an important role in building the personal project of students, demonstrating a high evaluation of family support for achieving educational goals. However, experience varies in relation to the characteristics of such support for individual decision and the pressure strategies or requirements for the achievement of obligations in higher education, showing significant differences according to socio-economic level of the families.
\end{abstract}

Keywords: Aspirations. Education-Work Transitions. High School Students. Parental Involvement. Parental Support.

\section{INTRODUCCIÓN}

La masificación de la educación superior y el fin del carácter elitista de este sistema educativo es uno de los rasgos más relevantes de las transformaciones ocurridas en la sociedad chilena en el tiempo reciente. En el período que cubre los últimos 15 años, el porcentaje de jóvenes entre los 18 y 24 años de edad que ingresó a estudiar una carrera de educación superior se incrementó desde un 15,6\% en 1990 a un 51,2\% correspondiente al año 2013. En la actualidad, a nivel nacional, la matrícula alcanza a más de 1200000 jóvenes y el porcentaje de participación de estudiantes pertenecientes a los dos quintiles más pobres de la sociedad se ha cuadruplicado en la última década, particularmente en el área de la educación técnica de nivel superior, que representa cerca del $45 \%$ del total de la matrícula. ${ }^{2}$

Aunque aún persiste una brecha significativa en el acceso a la educación postsecundaria considerando el nivel socioeconómico de la población, el actual proceso de expansión del sistema está mayormente concentrado en los estratos medios y bajos, por lo que es posible afirmar que se trata de cambios transversales que afectan 
al conjunto de la sociedad chilena (DONOSO, S.; DONOSO, G.; ARIAS, 2010; CARRASCO; ZÚÑIGA; ESPINOZA, 2014).

El impacto de este proceso es indiscutible y da cuenta de una transformación generacional de gran relevancia; un porcentaje cercano al 65\% de los actuales estudiantes, solo con el ingreso a una carrera de educación superior, ya ha logrado superar el nivel educacional de sus padres (CASTILLO; CABEZAS, 2010). La elaboración de un proyecto educativo postsecundario, la elección de una carrera profesional y un centro de estudios de nivel superior, así como también la consideración de los factores académicos y financieros que condicionan las decisiones sobre el futuro, constituyen, de este modo, rasgos extendidos en la construcción de expectativas de los jóvenes estudiantes en la actualidad, más allá de las diferencias socioeconómicas predominantes (ORELLANA, 2011; SEPÚLVEDA; VALDEBENITO, 2014). Pero la continuidad de estudios superiores representa también un desafío de financiamiento individual y familiar de enorme magnitud; no puede obviarse, en este plano, que el sistema de educación superior chileno es uno de los más onerosos del mundo, exigiendo un gran esfuerzo económico por parte de los estudiantes y su grupo familiar. El gasto privado en educación superior alcanza al 65\% del gasto total, y las carreras universitarias, en promedio, son las más largas en duración dentro del conjunto de los países miembros de la OCDE: $:^{3}$ 6,3 años en promedio respecto al 4,3 de promedio de los países miembros de esta organización ${ }^{4}$ (MELLER, 2011).

Todo lo anterior ha tornado compleja la transición de la educación secundaria a la educación superior, así como, en términos más generales, el proceso de transición del sistema educativo al mundo del trabajo, reconociéndose la extensión del período formativo que requieren las nuevas generaciones antes de alcanzar su independencia socioeconómica y el costo asociado a este proceso. Como consecuencia, este hecho ha implicado la prolongación en el tiempo de diversas modalidades de soporte familiar y el incremento de la responsabilidad financiera de los padres asociado al desarrollo de un proyecto educativo postsecundario de un número creciente de jóvenes. Pese a la relevancia de estas transformaciones, hasta ahora la investigación educativa ha otorgado una baja importancia a la familia y su grado de influencia en la experiencia de los jóvenes estudiantes, existiendo escasa investigación sobre los procesos de apoyo familiar a la construcción de expectativas educativo-laborales futuras de sus hijos y la definición y sostenimiento de los proyectos de mediano y largo plazo que estos elaboran (WYN; LANTZ; HARRIS, 2011). 
Aunque en la literatura relacionada a este tema abundan estudios que establecen una asociación directa entre las expectativas de los estudiantes y el capital cultural y socioeconómico de sus padres, por lo general se observa una preocupación marginal acerca de la manera cómo estos factores influyen en las opciones educativo-laborales que construyen los estudiantes a lo largo de su experiencia formativa (JONES; SCHNEIDER, 2009). De igual manera la investigación también es incipiente en analizar el rol que adquieren las expectativas de los padres en las decisiones de futuro y las dinámicas de apoyo y colaboración parental en el proceso de toma de decisiones en la etapa previa al egreso de la enseñanza secundaria (IRWIN; ELLEY, 2013).

Esta debilidad en el trabajo investigativo contrasta con la importancia creciente que se le asigna a la familia como soporte de las trayectorias educativas que siguen los jóvenes en la actualidad. En efecto, las ideas dominantes que han estado detrás del análisis de las expectativas y trayectorias educativas de las nuevas generaciones enfatizan enlaincidencia del proceso de individualización que caracteriza a la sociedad contemporánea (BECK, 1999; HEINZ, 2009). Desde esta perspectiva, el declive de algunas de las estructuras institucionales tradicionales, como ocurre con la escuela, ha incrementado la presión hacia los propios sujetos respecto a la necesidad de asumir una creciente responsabilidad en los logros y fracasos de su experiencia educativa, así como también en sus definiciones vocacionales de futuro; lo anterior ha incidido en un incremento de la demanda hacia las familias para la asunción de compromisos en la educación y socialización extracurricular de sus hijos, así como también servir de sostén, por un período prolongado de tiempo, ante la expansión de los años de estudio postsecundarios de un número ingente de jóvenes y las consecuencias socioeconómicas que este hecho acarrea (LÓPEZ BLASCO, 2006; TOGUCHI; BENGTSON, 2009).

La familiarización es el concepto que da cuenta de esteincremento de la responsabilidad socioafectiva y socioeconómica de padres u otros adultos significativos respecto a los jóvenes, y que constituye un pilar fundamental para el éxito o fracaso de los procesos educativos que experimentan las nuevas generaciones (ULE; ŽIVODER; DU BOIS-REYMOND, 2015). En la actualidad, los jóvenes no solo deben enfrentar un largo período de dependencia familiar, sino que también desarrollar estrategias respecto a la incertidumbre asociada a sus propias elecciones educativo-laborales y su sustentabilidad en el tiempo; esto significa, para una proporción creciente de familias, la necesidad de sostener e incrementar este soporte, toda vez que las 
transiciones se han vuelto, a la vez, más prolongadas, complejas e inciertas (FURLONG; CARTMEL; BIGGART, 2006).

Los estudios que han abordado el rol parental en el desarrollo de proyectos educativos postsecundarios de los jóvenes, dan cuenta de dos aspectos fundamentales que caracterizan el tiempo presente: por una parte, la constatación de un incremento de las expectativas de los padres para que sus hijos continúen los estudios superiores una vez finalizada la educación secundaria5 (CIDE, 2012) y, por otra, la disposición de los padres y/o el entorno familiar inmediato para realizar un esfuerzo material en pos del logro académico de sus hijos. En este marco, la educación es considerada un valor fundamental y el principal resorte de movilidad social destinado a alcanzar el objetivo de incremento de ingresos económicos y realización profesional de las nuevas generaciones (CASTILLO, 2013). Aunque estas características son reconocidas como elementos transversales en la experiencia de las actuales familias, preguntas acerca de la modalidad de acompañamiento padres-hijos en la toma de decisiones vocacionales, grado de incidencia parental en la definición de objetivos de futuro, nivel de conocimiento acerca del mercado educacional en la definición de objetivos de estudio, así como la negociación de espacios de autonomía en la extensión del período de dependencia estudiantil, establecen interrogantes de innegable interés para una agenda de investigación educativa en este ámbito, más aún en el marco de sociedades en las que las diferencias sociales y económicas constituyen un factor de distinción relevante de estas dinámicas familiares.

Este artículo intenta ser un aporte en esta dirección presentando antecedentes de un estudio en desarrollo sobre la trayectoria educativo-laboral de jóvenes estudiantes chilenos. En particular, el interés se focaliza en el análisis de las aspiraciones y proyectos educativos postsecundarios de estudiantes que cursaban su último año de formación secundaria a comienzos de la presente década, considerando el rol y la incidencia del soporte familiar en este proceso. Como podrá verse, el artículo discute acerca de las dimensiones particulares que asume lo que Leccardi (2010) denomina el pacto de solidaridad entre generaciones, y los procesos de negociación de un proyecto de autonomía individual en el marco de una experiencia de dependencia familiar prolongada a partir de la expansión de las alternativas educativas de los jóvenes en la actualidad. Se sostiene que el conocimiento sobre esta realidad permite un análisis más acabado del proceso de transición educativa de las nuevas generaciones, generando nuevas orientaciones y campos de intervención para las políticas educativas del tiempo presente. 


\section{ANTECEDENTES CONCEPTUALES}

La demanda por más educación y la ampliación de la cobertura de los niveles de enseñanza secundaria y postsecundaria constituyen rasgos predominantes en las sociedades modernas, cuestión que ha generado importantes transformaciones socioculturales en las últimas décadas. Uno de estos cambios tiene que ver con la mayor disposición de las familias a sostener por un período prolongado de tiempo la moratoria estudiantil de sus hijos y el conjunto de exigencias socioeconómicas asociadas a la continuidad de estudios de nivel superior y a la postergación de ingreso al mercado laboral (SELLAR; GALE; PARKER, 2011; GRYTNES, 2011). En efecto, algunos autores plantean que este hecho refleja la existencia de un nuevo paradigma en la sociedad actual, basado en el aumento de las expectativas educacionales bajo las reglas del orden social neoliberal y que tiene como consecuencia el incremento y extensión de la responsabilidad financiera de los individuos y sus familias en pos de lograr las certificaciones necesarias para una adecuada integración socioeconómica (JONES, 2009; WARD, 2012).

Esta característica es extensible al conjunto de la sociedad, observándose una disposición similar en aquellos sectores sociales que hasta hace algunas décadas no tenían acceso a mayores oportunidades formativas. Furlong y Cartmel (2007), al analizar la realidad británica, señalan que el creciente interés que las familias de clase trabajadora le otorgan a la educación se debe al debilitamiento de la dicotomía entre clase trabajadora y clase media en el ámbito productivo-laboral, así como también al surgimiento de un campo laboral más difuso, particularmente asociado al sector servicios, donde la división social es menos visible y, por tanto, mayormente abierto a la selección guiada por las credenciales educativas que pueden evidenciar los propios sujetos. La expansión del mercado educativo de la educación superior es el complemento institucional de este escenario, evidenciándose un crecimiento sostenido y diversificado de la oferta de este nivel educacional a nivel mundial, aunque con resultados discutibles en consideración al mejoramiento de las brechas de desigualdad al interior de los países (CARNOY et al., 2013).

Recientemente, estudios desarrollados en la línea de análisis de la subjetividad juvenil y de la transición o curso de vida de la población joven han permitido profundizar en la relación generacional que sustenta esta nueva realidad. Leccardi (2010) señala que, en el marco de las transformaciones socioeconómicas de las últimas décadas, ha surgido un nuevo pacto de solidaridad entre generaciones, el que incide en 
los procesos de negociación de un proyecto de autonomía individual en el marco de una experiencia de dependencia familiar prolongado, debido a la extensión del período destinado a la realización de estudios y la mayor complejidad del proceso de inicio y consolidación de la vida laboral. El elemento básico de este pacto radica en una lógica de interdependencia o desarrollo conjunto, que Scabiani, Marta y Lanz (2007) caracterizan como de protección flexible entre padres e hijos. Este proceso implica el reconocimiento mutuo y la adaptación de las relaciones al interior del entorno familiar a partir de la constatación de que el logro de la independencia absoluta se alcanza sobre la base de un vínculo de protección que se sostiene en el tiempo de manera mucho más evidente de lo que ocurría con anteriores generaciones. Con todo, se trata de un tipo de relación variable y que se define en el marco de un amplio campo de acuerdos y negociaciones que pueden transitar desde una mayor intercomunicación generacional hasta lo que Beck (1999) describió como una ignorancia reflexiva en este pacto o armisticio recíproco entre padres e hijos (LÓPEZ BLASCO, 2006).

Biggart y Walther (2006) señalan que, en un contexto de desestandarización de los procesos de transición educativa hacia el mundo del trabajo que experimentan los jóvenes en la actualidad, es un error reducir esta transición a una simple dicotomía entre dependencia y autonomía en la experiencia de los sujetos; para estos autores, parece necesario poner atención a la emergencia de una variedad de estados de semidependencia durante el período de transición educacióntrabajo, los que deben ser pesquisados y delimitados como parte de las biografías de las personas pertenecientes a las nuevas generaciones. En efecto, aunque ambos conceptos estén relacionados, dependencia se refiere fundamentalmente a una dimensión socioeconómica en la relación de los jóvenes con sus familias, particularmente asociada al financiamiento de estudios y/o sostenimiento material durante el tiempo destinado fundamentalmente a la realización de estudios postsecundarios; en cambio autonomía remite a cuestiones propiamente identitarias de los sujetos, tales como la libertad en la toma de decisiones sobre aspectos de la vida personal o el grado de consolidación de un estilo de vida independiente, que no dependen necesariamente de haber alcanzado una independencia económica y haber realizado una transición residencial desde el hogar paterno. La delimitación del carácter de esta relación y su manifestación en las distintas etapas del itinerario formativo de los jóvenes representan un ámbito de gran relevancia y permiten analizar de un modo más complejo los cambios educacionales en el mundo actual. 
Por otra parte, los estudios que han abordado el proceso de elección de educación superior dan cuenta de un fuerte contraste entre las decisiones que toman los jóvenes de estratos socioeconómicos bajos respecto a sus pares de clase media en este proceso. Aunque los estudios dan cuenta de manera consistente de la incidencia de la familia (o más directamente de los padres) en las elecciones y proyectos educativo-laborales de futuro, como se señala en investigaciones recientes, el análisis debería considerar el efecto diferenciado de esta influencia, incluyendo variables tales como el género o la clase social. En efecto, Reay, David y Ball (2005) observan diferencias en el campo de influencia de padres y madres en el proceso de toma de decisión educativa de sus hijos y señalan, además, que resulta necesario considerar diversos aspectos que pueden estar involucrados en esta relación: interés general, influencia y apoyo, financiamiento o intromisión en las decisiones de los jóvenes. Para estos autores, la consideración del habitus familiar, entendido como el sistema de perspectivas, experiencias y predisposiciones que están profundamente arraigados y que son compartidos por todos los miembros de la familia, constituye una perspectiva analítica fundamental para el análisis de las diferencias en el proceso de influencia familiar en la elección del futuro educacional de los jóvenes.

En este marco, otros estudios han analizado el rol de la familia en las elecciones de estudios superiores, considerando el crecimiento y homogenización de una nueva clase media en las sociedades modernas, asociadas a las transformaciones del mercado del trabajo y la ampliación de lo que Goldthorpe (1995) denomina la service class. Brooks (2003) destaca que, aunque los jóvenes de esta amplia nueva clase media compartan una base sociocultural de existencia común, pueden observarse diferencias considerables en el conocimiento que estos tienen del mercado de educación superior y los factores que privilegian para sus elecciones de futuro. En un estudio realizado en el Reino Unido, esta autora destaca que los jóvenes elaboran jerarquías de intereses distintos y valoran de un modo variable las instituciones de educación superior; en este proceso, la familia y el grupo de pares ejercen una influencia significativa en la definición de proyectos educativos futuros, resultando muchas veces determinante esta influencia en la viabilidad y plausibilidad de las opciones tomadas. Sobre esta base, es posible sostener que esta perspectiva de estudio resulta de gran importancia para una revisión analítica de los cambios vertiginosos que han estado asociados al crecimiento de la matrícula de educación superior en Chile y países similares. Una mirada más amplia 
de los factores que están asociados a las elecciones educativas de las nuevas generaciones resulta necesaria, más aún si se considera la escasa transparencia y desregulación que caracteriza a este mercado educativo.

\section{CONSIDERACIONES METODOLÓGICAS}

El material empírico sobre el que se organiza este artículo proviene del levantamiento de la investigación en marcha "Expectativas, proyectos educativo-laborales y trayectorias posegreso de jóvenes estudiantes secundarios: un estudio en la Región Metropolitana", que se realiza con el apoyo de FONDECYT. ${ }^{6}$ Este estudio, de carácter longitudinal, tiene como objetivo analizar los modelos de trayectoria educativo-laboral que derivan de la experiencia de una muestra de jóvenes estudiantes durante el período posterior a su egreso de la enseñanza secundaria. El estudio consiste en la aplicación de olas sucesivas de encuestas y entrevistas cualitativas a jóvenes que cursaban su último año escolar durante el año 2011 en distintos establecimientos educacionales de la Región Metropolitana de Santiago. Con posterioridad han existido cuatro etapas periódicas de levantamiento de información entre los años 2012 y 2015, recogiendo antecedentes sobre la situación posegreso de los jóvenes. En este marco, está prevista una última etapa de levantamiento de información durante el año 2016, cubriendo la experiencia de cinco años posteriores al egreso de la enseñanza secundaria de la muestra de jóvenes. En la primera fase del estudio, se implementó una encuesta dirigida a una muestra de estudiantes del último año de enseñanza secundaria pertenecientes a diversos tipos de establecimientos educacionales de la ciudad de Santiago y localidades adyacentes. El trabajo de campo para esta primera parte del estudio contempló la aplicación de un instrumento estructurado a 1888 jóvenes estudiantes de $4^{\circ}$ año medio (último año de educación secundaria) en 69 establecimientos educativos. ${ }^{7}$

En la construcción de esta muestra se consideró la dependencia administrativa de los centros educacionales seleccionados y la modalidad de estudios de los jóvenes encuestados (modalidad científico-humanista, EMCH, y técnico-profesional, EMTP) ${ }^{8}$. El procesamiento de los resultados incluyó también la consideración del nivel socioeconómico de los establecimientos participantes del estudio. Esta fue construida en base a la caracterización que establece el Ministerio de Educación chileno a través de la prueba nacional 
de medición de resultados escolares y que diferencia a los centros educacionales en relación a la situación socioeconómica promedio de las familias asociadas a cada establecimiento. ' Los resultados fueron complementados con material empírico de tipo cualitativo recogido a través de entrevistas individuales y grupos de discusión con jóvenes incluidos en la muestra. ${ }^{10}$ Aunque la investigación en desarrollo tiene por objetivo analizar los modelos de trayectorias de los jóvenes egresados de la enseñanza secundaria, los antecedentes en que se centra este artículo corresponden a un set de preguntas contenidas en las encuestas destinadas a indagar las percepciones de los estudiantes respecto al soporte familiar en su experiencia formativa y el grado de apoyo que estos perciben en la elaboración y consecución de sus proyectos educativos postsecundarios, aspectos que también han sido profundizados en la indagación cualitativa. Los datos recogidos mediante encuestas que se presentan en este artículo han sido sometidos a un análisis estadístico simple, con fines propiamente descriptivos y aplicado sobre los indicadores relevados en la indagación. Este proceso fue realizado para describir el comportamiento de tales indicadores en la muestra del estudio, identificar la concentración y desconcentración de datos, además de identificar la cantidad de casos agrupados para cada categoría de respuesta. El análisis de la información cualitativa, por su parte, estuvo orientado a caracterizar el discurso predominante en los actores en sus respectivos contextos, para luego elaborar una interpretación global de la información. El análisis del material se organizó a partir de los principios básicos de la teoría fundamentada e incluyó procedimientos de codificación temática con el objetivo de observar una eventual distribución social de las perspectivas sobre los temas de interés investigativo. El procedimiento de análisis del material comprendió la creación de categorías temáticas que tuviesen propiedades comunes, y la integración analítica de tales categorías con el objetivo de organizar los componentes buscando su coherencia interna e integración conceptual, para finalmente alcanzar la estructuración de un modelo comprensivo, construido a partir un discurso de discursos. Con ello se ha intentado entregar respuestas interpretativas a aquellos aspectos de interés acerca de la percepción, juicio y descripción del soporte familiar al desarrollo del proyecto personal de los jóvenes, lo que corresponde al foco principal de este artículo. ${ }^{11}$ Para el tratamiento del material, se trabajó con el soporte del programa computacional Atlas.ti. 


\section{PRESENTACIÓN DE RESULTADOS}

Los principales aspectos que se consideran en la presentación de resultados de este estudio remiten al reconocimiento de: (a) las altas aspiraciones de emprender la realización de estudios superiores que evidenciaban los jóvenes estudiantes al finalizar su enseñanza secundaria y el grado de cumplimiento de estas en las etapas sucesivas de sus respectivas trayectorias; (b) la percepción general que se recoge entre éstos acerca del apoyo o soporte familiar a sus proyectos educativos durante el período posterior a su egreso de la enseñanza obligatoria, y las diferencias observadas respecto a las características de acompañamiento y relación con el entorno familiar considerando el nivel socioeconómico de las familias; y (c) el grado de autonomía que la gran mayoría de los jóvenes señala tener en la actual etapa de sus vidas, y la distinción que puede realizarse entre esta dimensión propiamente identitaria y la dependencia material prolongada de padres u otros familiares relevantes, que incluye la permanencia en el hogar familiar por un período de tiempo significativo.

Respecto a la construcción de proyectos de futuro de los jóvenes objeto de esta investigación, la indagación ratifica la existencia de un alto nivel de aspiraciones por realizar estudios de educación superior, independientemente de la modalidad de estudios cursados, el rendimiento y logros escolares obtenidos y el nivel socioeconómico de sus familias. Sólo un porcentaje cercano al 11\% de los encuestados en este estudio señalaba, al año siguiente de finalizada su enseñanza media, su disposición a incorporarse al mundo del trabajo de manera inmediata, sin que existiese en su horizonte de futuro el interés por continuar estudios postsecundarios. El mayor porcentaje de quienes se encontraban en esta posición correspondía a jóvenes de los estratos socioeconómicos bajo y medio-bajo, en los que la proporción de casos se aproximaba al 20\% de este segmento. Casi la mitad de los jóvenes indicaba que su proyecto personal estaba orientado a terminar una carrera universitaria ${ }^{12}$ (tendencia que se eleva al 90\% de los casos de los estudiantes del nivel socioeconómico alto), mientras que un porcentaje cercano al $20 \%$ de la muestra señaló su interés por cursar estudios en una carrera técnica de nivel superior ${ }^{13}$ (siendo predominante el porcentaje de casos que señala esta alternativa en el estrato socioeconómico bajo y medio-bajo).

Aunque el proyecto de continuar estudios superiores constituye una realidad extendida entre los jóvenes de esta investigación, existen diferencias importantes a considerar cuando esta aspiración se sitúa en un plano temporal. En efecto, un tercio de los encuestados 
de los estratos medio-bajo y bajo señaló que la perspectiva de continuar estudios podría estar mediada por una etapa previa de ingreso al mercado laboral con el objetivo de reunir recursos para el financiamiento de sus estudios. Esta perspectiva desaparece en los estratos medio-alto y alto, en los que la transición a la educación superior se percibe como un proceso fundamentalmente de tipo lineal y las consideraciones de financiamiento de los estudios y otros gastos asociados a esta condición son asumidos como una situación no problemática al interior de su entorno familiar.

Más allá de esta distinción, por cierto relevante, el seguimiento de la trayectoria de los jóvenes ratifica la predominancia de la educación superior en la experiencia personal de la gran mayoría de los participantes en el estudio. Casi la mitad de la muestra, el $47 \%$ de los jóvenes, ingresó a un centro de educación superior al año siguiente de la finalización de su formación secundaria, mientras que, dos años después de su egreso, un poco más del 60\% de los jóvenes se encontraba cursando una carrera de educación de nivel terciario (de estos, cerca de un $20 \%$ reporta combinar sus estudios con una actividad laboral complementaria). Las Tablas 1 y 2 resumen lo anteriormente señalado; la indagación acerca de las aspiraciones iniciales da cuenta de una alta proporción de jóvenes que se proponía ingresar a la educación superior con posterioridad a su egreso de la enseñanza secundaria. Dos años después, dos tercios de los encuestados se encontraban estudiando una carrera de educación superior o en un proceso de combinación de actividades formativas y laborales, mientras que el tercio restante (aunque con una proporción mucho mayor entre los jóvenes pobres) tenía como actividad principal el desarrollo de una actividad remunerada. ${ }^{14}$

Tabla 1 - Manifestación de proyecto educativo-laboral de los estudiantes al egreso de la enseñanza secundaria por nivel socioeconómico (n 1888 casos)

\begin{tabular}{|l|c|c|c|c|c|}
\hline & Bajo & $\begin{array}{c}\text { Medio- } \\
\text { bajo }\end{array}$ & Medio & Medio-alto & Alto \\
\hline Trabajar y no estudiar & $17.9 \%$ & $22 \%$ & $7.9 \%$ & $4.9 \%$ & $1.4 \%$ \\
Estudiar una carrera universitaria & $22.6 \%$ & $26.7 \%$ & $42.1 \%$ & $68.7 \%$ & $88.5 \%$ \\
Estudiar una carrera técnica superior & $39.3 \%$ & $24.8 \%$ & $22.3 \%$ & $10.6 \%$ & $3.4 \%$ \\
Estudiar una carrera militar o policial & $1.2 \%$ & $3 \%$ & $2.9 \%$ & $2.4 \%$ & $0.0 \%$ \\
Ni estudiar ni trabajar & $1.2 \%$ & $1.2 \%$ & $0.8 \%$ & $0.0 \%$ & $1.4 \%$ \\
Otro ${ }^{15}$ & $17.8 \%$ & $22.3 \%$ & $24 \%$ & $13.4 \%$ & $5.3 \%$ \\
\hline
\end{tabular}

Fuente: Sepúlveda y Valdebenito, 2014 
TABLA 2 - Situación educativo-laboral de los estudiantes al segundo año de egreso de la enseñanza secundaria por nivel socioeconómico (n 1011 casos)

\begin{tabular}{|l|c|c|c|c|c|c|}
\hline & Bajo & Medio-bajo & Medio & Medio-alto & Alto & Total \\
\hline $\begin{array}{l}\text { Trabajo } \\
\text { remunerado }\end{array}$ & $51.5 \%$ & $44.9 \%$ & $26.8 \%$ & $8.9 \%$ & $0.0 \%$ & $28.5 \%$ \\
$\begin{array}{l}\text { Estudios } \\
\text { universitarios }\end{array}$ & $13.4 \%$ & $15.2 \%$ & $29.7 \%$ & $67.7 \%$ & $92.2 \%$ & $39.3 \%$ \\
$\begin{array}{l}\text { Estudios técnico- } \\
\text { superiores }\end{array}$ & $19.6 \%$ & $23.2 \%$ & $33.2 \%$ & $13.5 \%$ & $5.4 \%$ & $20.6 \%$ \\
$\begin{array}{l}\text { Otras } \\
\text { actividades }\end{array}$ & $15.5 \%$ & $16.7 \%$ & $10.3 \%$ & $9.9 \%$ & $2.4 \%$ & $11.6 \%$ \\
\hline
\end{tabular}

Fuente: Elaboración propia a partir de resultados del estudio

Una primera constatación, de este modo, ratifica la predominancia de la continuidad de estudios en la trayectoria de los jóvenes luego de finalizada su enseñanza secundaria. Esta realidad puede ser aún más extendida; estudios recientes señalan el aumento de jóvenes que se incorporan a la educación superior (muy particularmente en carreras de educación técnica) luego de algunos años de desempeño en una actividad laboral; en no pocos casos, la elección formativa corresponde a una decisión orientada por el tipo de actividad o trabajo realizado previamente, por lo que la elección tiende a alcanzar un mayor apego a una decisión de tipo vocacional (SEPÚLVEDA; UGALDE, 2010). Más allá de lo anterior, para el cumplimiento de este objetivo de continuidad de estudios, el soporte familiar adquiere un rol importante no solo de sostén económico durante un período de tiempo prolongado, sino que también de apoyo y adscripción al proyecto educativo que los jóvenes construyen. Como consecuencia, un segundo aspecto relevante de análisis, desde este punto de vista, tiene que ver con la percepción de los jóvenes en estudio acerca del soporte familiar existente durante el período actual de sus vidas y las características que este tiene.

Respecto a esta situación, los antecedentes recogidos en la indagación señalan que los jóvenes destacan a su núcleo familiar (independientemente de su composición o características particulares) como el ámbito más importante de desarrollo de relaciones de sociabilidad, percepción de confianza y apoyo a sus proyectos personales. Para los jóvenes que estudian, la familia es su principal soporte financiero y emocional, y sus perspectivas de independencia se 
proyectan en un futuro relativamente lejano, asociado a la finalización de sus estudios postsecundarios. Los jóvenes que trabajan también tienen a la familia como un referente principal, y, en un porcentaje importante de los casos, señalan que los principales motivos que los llevan a iniciar una vida laboral tienen que ver con la necesidad de entregar un aporte al sustento de su grupo inmediato. La percepción de que existe un fuerte apoyo familiar para la continuidad de estudios fue un aspecto ampliamente recogido en las entrevistas realizadas en el marco de esta investigación. El reconocimiento al esfuerzo financiero asociado a este desafío es una cuestión ampliamente relevada entre los estudiantes que provienen de familias con mayores dificultades económicas y que se refleja en los siguientes testimonios:

Lo importante aquí es que la familia lo esté apoyando a uno, que si uno toma una decisión sobre los estudios y la familia no quiere y no lo apoya a uno, como que se echa para abajo al tiro [...]; por ejemplo, cuando un hombre quiere estudiar algo como del área de música, los papás pueden ser muy machistas y dicen que no, entonces, al no apoyarlo, el hijo se va a frustrar y después no va a ser nada y ahí puede quedar vagando y sin futuro después. En cambio, si los papás siempre están diciendo "a mí no me importa lo que elijas tú, pero yo siempre te voy a estar apoyando", uno se siente orgulloso de eso, entonces, le dan más ganas de seguir estudiando y lograr esa meta para demostrar que uno vale en verdad; mis papás siempre me han dicho que cuente con su apoyo y que elija estudiar algo en que me sienta realizada [...]. (Mujer, NSE medio-bajo).

Mi mamá me dijo que, si es necesario, se va a esforzar para que yo pueda sacar lo que quiero; obviamente yo encuentro mejor eso, porque claro, los créditos después hay que pagarlos y se termina pagando mucho más de lo que uno pidió; entonces, si mi mamá me va a pagar esto, yo me voy a esforzar lo suficiente para que, cuando tenga la plata, yo pueda devolverle lo que ella gastó en mí, ayudando a la casa para pagarles lo que ellos hicieron por mí [...]. (Hombre, NSE medio).

A mí, en mi familia, me dijeron que no pidiera crédito cuando estudiara en la universidad. Yo tengo otra hermana que también está estudiando, tuvieron que pagarle los dos primeros meses completa la carrera, pero ahora ella es becada, tiene dos becas que cubren casi todos los gastos de su carrera. Yo no sé a qué puedo postular yo, pero mis papás me dijeron que no, que no usara crédito, que no querían que yo después estuviera pagando mucho más de lo que pedí [...], me dijeron que no trabajara, ni que optara a crédito. Que yo me dedicara a mis estudios y que ellos me ayudarían [...] yo tengo un hijo, así que igual me ayudan mucho. (Mujer, NSE medio-bajo).

El alto costo que implica estudiar una carrera de educación superior es una situación que se encuentra presente de manera patente en el discurso de los jóvenes pertenecientes a los estratos socioeconómicos medios y bajos, y surge constantemente como una referencia que condiciona las opciones personales en la elección de 
carreras o centros de estudios. La disposición de apoyo financiero, más allá de las dificultades que esto implica, es un rasgo predominante en la experiencia de estos jóvenes y sus familias, y ratifica el carácter universal del reconocimiento de la educación como un factor de inserción social y mejoramiento de las condiciones de vida. Lo anterior también tiene implicancias en las decisiones personales de los jóvenes, existiendo mecanismos de autopresión que se evidencian en los discursos individuales:

Yo vivo solamente con mi madre y no tengo ningún hermano mayor; ella me pregunta todos los días qué quiero hacer y todo eso. Yo creo que también es un reto para ella, que es la primera vez que tiene que pensar en la universidad; la mayor parte de los gastos, si yo quisiera estudiar, correrían por parte de ella, entonces, ella también se tiene que planificar y todo eso, y ve todas las cosas encima, yo encuentro que es razonable que ella me pregunte todos los días si quiero estudiar o no. Igual por parte de mi papá, siempre me ha dicho que estudie lo que sea, pero que sea la mejor; lo que yo quiera me va a apoyar; aunque me insisten en la importancia que estudie algo que me de la seguridad, estabilidad, que sea una decisión que valga la pena [...]. (Mujer, NSE medio-bajo).

Mis papás me han estado inculcando siempre hacia la medicina, desde pequeño me han mostrado lo del cuerpo humano, me explican con sus conocimientos y por láminas y por todo tipo de cosas. Ellos son panaderos y yo creo que buscan que yo tenga una vida menos sacrificada que la que ellos han tenido, para darme las cosas que yo necesito. Ellos se han sacrificado todo este tiempo; me han pagado el estudio, me pagan profesor particular para que me prepare, me pagan clases de inglés, biología, matemáticas, todas las que necesito para poder salir adelante. Más que por la preparación, porque sé que tengo los conocimientos, los tengo y estoy seguro de ello, lo que pasa es que yo soy un poco nervioso y tengo temor que los nervios me traicionen en la prueba y me quede en blanco. Ese sería mi mayor temor, no responder a su esfuerzo [...]. (Hombre, NSE medio-bajo).

Yo siento presión de parte de mi familia, porque soy el menor, mi hermano salió de la universidad y tiene título, mi hermana salió de la universidad y tiene título. Y soy el que queda, entonces no puedo ser menos que ellos, no puedo ser la oveja negra, no puedo quedarme atrás, yo creo que las circunstancia en las que vivo me llevan a no quedarme atrás. Y cada vez tratar de mejorar; por ejemplo, a mi hermana le fue mejor que a mi hermano, entonces a mí me dicen "a ti te tiene que ir bien". Y mis papás supuestamente, cuando me hablan, me dicen "no, yo no te presiono ni nada, pero el próximo año tienes que estar estudiando". Entonces igual es una presión, no se siente tanto, pero yo sé que quieren que yo estudie y que responda a sus esperanzas. (Hombre, NSE medio).

La constatación de que no se puede errar en una elección vocacional de educación superior debido al alto costo socioeconómico que esto implica, es una afirmación fuertemente arraigada en el discurso predominante de los jóvenes. Corresponde a un campo de responsabilidad 
personal de cada individuo y que se articula con el compromiso adquirido con los padres u otros familiares relevantes al momento de emprender la empresa de continuidad de estudios de nivel superior.

En este marco, con todo, es posible observar diferencias relevantes cuando se interroga a los jóvenes acerca de las características que asume el apoyo familiar más allá del sostenimiento propiamente financiero de los estudios postsecundarios. En efecto, en nuestra investigación, fue posible observar diferencias en la percepción del apoyo informativo y sugerencias de opciones de estudio que puede aportar el entorno familiar a estos jóvenes. Particularmente en las respuestas recogidas en la etapa de finalización de su formación secundaria, los estudiantes pertenecientes a familias de nivel socioeconómico bajo y medio-bajo reconocían una situación de orfandad respecto a esta dimensión. Junto con lo anterior, otro aspecto que destaca del análisis del soporte familiar en la experiencia de los jóvenes está asociado a la distinción que puede hacerse entre apoyo y presión para el cumplimiento de los objetivos personales.

Tal como puede observarse en la Tabla 3, los jóvenes de las familias de estrato socioeconómico bajo manifiestan una menor prevalencia de esta última dimensión, cuestión que contrasta con las respuestas que se recogen de los grupos medios de este estudio. En efecto, en estos últimos, es posible identificar un mayor reconocimiento de que el apoyo familiar está asociado también a una conducta de seguimiento y presión para el cumplimiento de las obligaciones conducentes a los objetivos personales. La presión corresponde aquí a una actitud o disposición de los adultos significativos que puede expresarse de múltiples formas: un mayor conocimiento y acciones de seguimiento de la vida estudiantil de los jóvenes; solicitud de información y actividades de control ante obligaciones académicas y procesos evaluativos; seguimiento de rutinas y hábitos cotidianos de los jóvenes en esta nueva etapa de la vida estudiantil, entre otras acciones. Este aspecto es ampliamente recogido en el material cualitativo de la investigación, dando cuenta de un rasgo sociocultural novedoso en el tiempo reciente. ${ }^{17}$ 
TABLA 3 - Percepción respecto de la importancia y soporte de la familia (\% Muy de acuerdo + De acuerdo de respuestas); tercera medición estudio longitudinal

\begin{tabular}{|l|c|c|c|c|c|}
\hline & Bajo & $\begin{array}{c}\text { Medio- } \\
\text { bajo }\end{array}$ & Medio & $\begin{array}{c}\text { Medio- } \\
\text { alto }\end{array}$ & Alto \\
\hline $\begin{array}{l}\text { En general, siento que mi familia me } \\
\text { apoya en lo que hago }\end{array}$ & $80.4 \%$ & $92.6 \%$ & $97.6 \%$ & $94 \%$ & $94.9 \%$ \\
$\begin{array}{l}\text { A mi familia le gustaría que hiciese } \\
\text { otra cosa de lo que deseo }\end{array}$ & $20 \%$ & $26.1 \%$ & $17.1 \%$ & $12.6 \%$ & $15.4 \%$ \\
$\begin{array}{l}\text { En mi familia se preocupan y me presionan } \\
\text { para que cumpla con mis responsabilidades } \\
\text { En mi familia no necesariamente entienden } \\
\text { mis intereses y mis gustos }\end{array}$ & $24 \%$ & $62.3 \%$ & $64.3 \%$ & $62.1 \%$ & $45.3 \%$ \\
$\begin{array}{l}\text { En mi familia me dan bastante libertad } \\
\text { para desarrollar lo que yo quiero hacer }\end{array}$ & $74 \%$ & $90 \%$ & $37 \%$ & $31 \%$ & $27.3 \%$ \\
\hline
\end{tabular}

Fuente: Elaboración propia a partir de resultados del estudio

Brooks (2003) observó una realidad similar para el caso británico en el marco de las transformaciones sociales de las últimas décadas. Como se señaló anteriormente, la autora aborda las características de una amplia clase media en la sociedad actual y, muy particularmente, una clase media liminal que se distancia en sus aspiraciones y conductas respecto a la tradicional clase obrera (como también, obviamente, de los sectores de mayores ingresos de la sociedad). En su análisis, una distinción relevante para un grupo que tiende a manifestar una homogenización de tipo sociocultural (asociado a la pertenencia a la service class) radica, por una parte, en el grado de conocimiento y vinculación con el mercado de educación superior existente en el entorno familiar y, por otra, en las orientaciones y disposiciones de apoyo del grupo familiar en la toma de decisiones y trayectorias que experimentan los jóvenes una vez que finalizan su educación obligatoria. Este aspecto, el capital sociocultural de las familias en el marco de una tendencia a la homogenización socioeconómica, parece ser cercano a lo reseñado en nuestro estudio y resulta un insumo sugerente para el análisis de las orientaciones de acción y las trayectorias educativo-laborales efectivas que realizan los jóvenes. Un mayor conocimiento de esta realidad permitiría, por cierto, la orientación de políticas consistentes ante el cuadro de masificación de la educación superior que hoy experimentan países como el nuestro. 
Finalmente, un tercer elemento relevante que surge de esta investigación tiene que ver con la perspectiva de largo plazo y el lugar de la familia de origen en la construcción del proyecto personal de los sujetos. En el discurso de los jóvenes, la mirada crítica o distante de la influencia familiar en la autonomía personal es marginal, sin que se evidencien distinciones relevantes por nivel socioeconómico, género o modalidad de estudios; de manera transversal, se asume que la consecución del objetivo de alcanzar la concreción de estudios superiores se hace, necesariamente, dentro del entorno familiar y bajo el sostén económico y emocional de la familia, ratificando la noción de pacto de solidaridad de Leccardi (2010). En los testimonios personales, llama la atención la marcada valoración del hogar familiar como un refugio personal y la minimización de las relaciones conflictivas intergeneracionales que, aunque existentes (y en algunos casos muy agudas), no alcanzan la relevancia necesaria para constituirse en un foco de conflicto axial como se destacaba en la literatura sobre juventud en otros períodos históricos.

En efecto, en la proyección de los itinerarios de vida que construyen los jóvenes, es justamente el abandono del espacio familiar el que se posterga para el cumplimiento de sus objetivos de estudio y el hito que se reconoce como fundamental en la condición de bacerse adulto. Antes que comenzar a trabajar, o la finalización del período de estudios, es la independencia del entorno inmediato lo que emerge como el hito más significativo del cambio de vida o transición a la vida adulta. El Gráfico 1 da cuenta de las respuestas de los encuestados respecto a este tema, evidenciando incluso el aumento de la proporción de respuestas que sitúan en este paso el principal marcador de tránsito hacia la adultez. De este modo, es posible identificar, en la visión de la gran mayoría de los jóvenes, una diferencia marcada entre la independencia del hogar familiar y la autonomía de su vida personal (que en términos amplios puede entenderse como la libertad para tomar sus propias decisiones). De hecho, más de mitad de los encuestados en el primer año de egreso de la educación secundaria señala que en ese momento ya había logrado un nivel de autonomía plena respecto a sus padres u otros familiares significativos, cuestión que se extiende a ámbitos tales como las relaciones de pareja y la administración del tiempo en su vida cotidiana. 
GRÁFICO 1 - ¿Cuándo una persona es adulta? Variación percepción 2011-2013

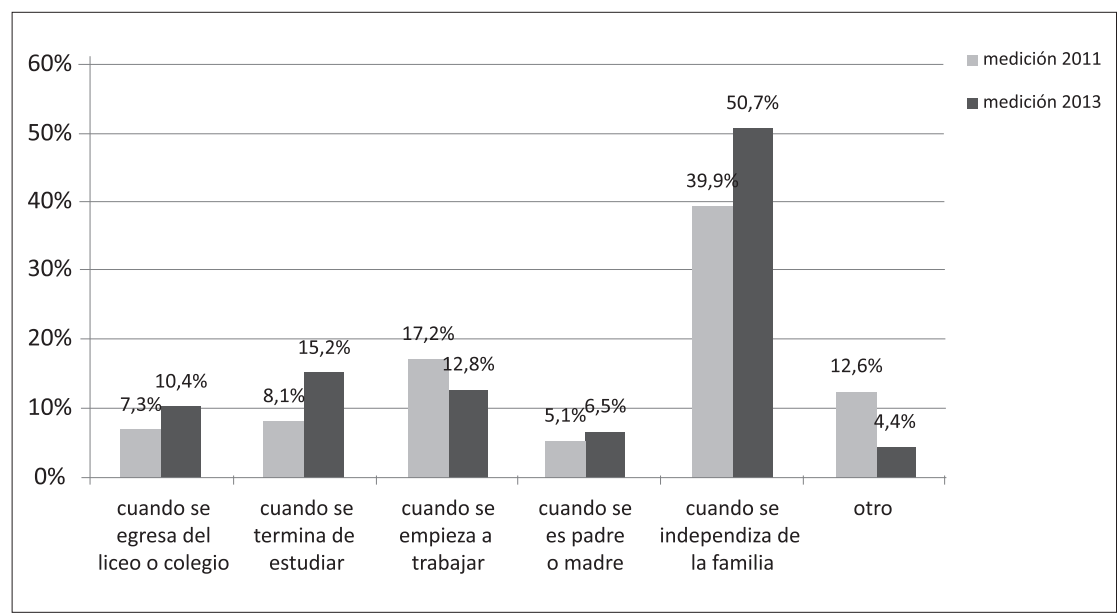

Fuente: Elaboración propia a partir de resultados del estudio

Así, la extensión del período de dependencia se constituye como un rasgo dominante para las nuevas generaciones que se incorporan de manera masiva a la educación superior, aunque este hecho se distingue de la autonomía personal, aspecto que es ampliamente reconocido por los jóvenes, independientemente del nivel socioeconómico, género o actividad principal que se encuentren realizando.

\section{CONCLUSIONES}

En síntesis, los resultados de este estudio evidencian que:

(a) las aspiraciones de estudios superiores son extendidas entre los jóvenes, independientemente de su nivel socioeconómico, aunque, sin embargo, se observan diferencias importantes al analizar las opciones efectivas de ingreso a educación superior y la temporalidad considerada para su logro. Es posible identificar la emergencia de diversas racionalidades que darían cuenta de un ajuste de las aspiraciones y los itinerarios previstos para alcanzar los objetivos personales fuertemente condicionados por el nivel socioeconómico de origen. El seguimiento de la trayectoria de los jóvenes en estudio, en términos generales, da cuenta de un porcentaje significativo de casos en que efectivamente ellos ingresan a la educación superior, aunque muchos de estos lo realizan de un modo diferido en el tiempo y sobre la base de la combinación de estudios con actividades laborales; 
(b) en este marco, de manera general, el entorno familiar (u otros adultos significativos) juega un rol importante en la construcción del proyecto personal de los jóvenes, existiendo una alta valoración del apoyo otorgado para el logro de los objetivos personales. Lo anterior, sin embargo, contrasta con la existencia de diferencias en el soporte de información y orientación para las decisiones individuales, como también, de manera muy particular, en las estrategias de presión o exigencias para el cumplimiento de obligaciones y metas. Se observan diferencias importantes en este plano cuando el análisis considera el nivel socioeconómico de las familias de origen; junto a lo anterior, es posible hipotetizar la existencia de estrategias diferenciadas para alcanzar el objetivo de ingreso a la educación superior que puede tener consecuencias relevantes en el logro alcanzado y el grado de satisfacción personal de los jóvenes estudiantes;

(c) finalmente, los antecedentes empíricos también dan cuenta de los rasgos socioculturales predominantes en el discurso de los jóvenes acerca de los procesos de autonomía individual en un contexto de dependencia familiar prolongado, observándose una clara distinción entre ambas dimensiones en las trayectorias biográficas de esta generación social; lo anterior es asumido de un modo no conflictivo por la gran mayoría de los jóvenes y naturalizado como un rasgo característico de la experiencia de vida de este grupo etario.

En síntesis, estos resultados dan cuenta de rasgos socioculturales específicos de las nuevas generaciones que, de manera creciente, continúan los estudios superiores una vez finalizada su enseñanza secundaria. El rol estratégico de los padres u otros familiares relevantes en este proceso representa una constatación que debería tenerse en consideración para el desarrollo de políticas públicas en el ámbito educativo. Como puede desprenderse de la literatura reciente y como los datos empíricos de este estudio también corroboran, la familia y el soporte que esta representa para el desarrollo de los proyectos educativos de sus hijos se han transformado en un factor fundamental que incide en el éxito o fracaso de esta empresa y, por tanto, las políticas no deberían obviar su incidencia en la actualidad y hacia el futuro.

\section{REFERENCIAS}

ANDRES, L.; WYN, J. The making of a generation: the children of the 1970s in adulthood. Toronto: Toronto University Press, 2010.

BEADLE, S.; HOLDSWORTH, R.; WYN, J. (Orgs.). For we are young and...? Young people in a time of uncertainty. Melbourne: Melbourne University Press, 2011. 
BECK, U. Democratización de la familia. In: (Org.). Hijos de la libertad. México: Fondo de Cultura Económica, 1999. p.187-209.

BIGGART, A.; WALTHER, A. Coping with Yo-Yo Transitions. Young adult's struggle for support, between family and state in comparative perspective. In: LECCARDI, C.; RUSPINI, E. (Orgs.). A New Youth? Young people, generations and family life. London: Ashgate, 2006. p.41-62.

BROOKS, R. Young people's higher education choices: the role of family and friends. British Journal of Sociology of Education, London, v. 24, n. 3, p. 283-297, June. 2003.

CARNOY, M. et al. University expansion in a changing global economy: triumph of the BRICs? Stanford: Stanford University Press, 2013.

CARRASCO, E.; ZÚÑIGA, C.; ESPINOZA, J. Elección de carrera en estudiantes de nivel socioeconómico bajo de universidades chilenas altamente selectivas. Calidad en la Educación, Santiago, n. 40, p. 95-128, jul. 2014.

CASTILLO, J. Movilidad social y educación en Chile. In: TEZANOS, J. (Org.). Juventud, cultura y educación: perspectivas comparadas en España y Chile. Madrid: Siglo XXI Editores: Biblioteca Nueva, 2013. p. 267-286.

CASTILLO, J.; CABEZAS, G. Caracterización de jóvenes primera generación en educación superior: nuevas trayectorias hacia la equidad educativa. Calidad en la Educación, Santiago, n. 32, p. 44-76, jul. 2010.

CENTRO DE INVESTIGACIÓN Y DESARROLLO DE LA EDUCACIÓN - CIDE. IX Encuesta a actores del sistema educativo, Santiago, 2012. Disponible en: <www.cide. cl/documentos/Informe_IX_Encuesta_CIDE_2012.pdf $\geq$. Consultado el: 10 nov. 2014.

DONOSO, S.; DONOSO, G.; ARIAS, O. Iniciativas de retención de estudiantes en educación superior. Calidad en la Educación, Santiago, n. 33, p. 15-61, dic. 2010.

FURLONG, A.; CARTMEL, F. Graduates from disadvantaged families: early labour market experiences. London: Joseph Rowntree Foundation, 2005.

Young people and social change: new perspectives. London: Open University Press:-McGraw-Hill, 2007.

; BIGGART, A. Choice biographies and transitional linearity: Re-conceptualising modern youth transitions. Papers Revista de Sociología, Barcelona, v. 79, p. 225-239, enero 2006.

GOLDTHORPE, J. The service class revisited, In: T. BUTLER \& M. SAVAGE (Eds.) Social Change and the Middle Classes, London, UCL Press, 1995.

GRYTNES, R. Making the right choice! Inquiries into the reasoning behind young people's decisions about education. Young, Oslo, v.19, n. 3, p. 333-351, July. 2011.

HEINZ, W. Youth transitions in age of uncertainty. In: FURLONG, A. (Ed.) Handbook of youth and young adulthood: new perspectives and agendas. London: Routledge, 2009. p.3-13.

IRWIN, S.; ELLEY, S. Parents' hopes and expectations for their children's future occupations. The Sociological Review, Leicester, v. 61, n. 1, p. 111-130, Feb. 2013.

JONES, G. From paradigm to paradox: parental support and transitions to independence. In: SCHOON, I.; SILBEREISEN, R. Transitions from school to work: globalization, individualization, and patterns of diversity. Cambridge: Cambridge University Press, 2009.

JONES, N.; SCHNEIDER, B. The influence of aspirations on educational and occupational outcomes. In: FURLONG, A. (Ed.) Handbook of youth and young adulthood: new perspectives and agendas. London: Routledge, 2009. p. 392-398. 
LECCARDI, C. La juventud, el cambio social y la familia: de una cultura "de protección” a una cultura "de negociación”. Revista de Estudios de Juventud, Madrid, n. 90, p. 33-42, sep. 2010. LÓPEZ BLASCO, A. La familia como respuesta a las demandas de individualización: ambivalencias y contradicciones. Papers, Revista de Sociología, Barcelona, v. 79, p. 263284, enero. 2006.

MELLER, P. Universitarios, ¡el problema no es el lucro sino el mercado!. Santiago: Uqbar Editores, 2011.

ORELLANA, V. Nuevos estudiantes y tendencias emergentes en la Educación Superior: una mirada al Chile de mañana. In: JIMÉNEZ, M.; LAGOS, F. (Orgs.). Nueva geografía de la educación superior y de los estudiantes: una cartografía del sistema chileno, su actual alumnado y sus principales tendencias. Santiago: Ediciones Universidad San Sebastián, 2011, p. 80-142.

REAY, D.; DAVID, M.; BALL, S. Degrees of choice: social class, race and gender in higher education. London: Trenham Books, 2005.

SCABIANI, E.; MARTA, E; LANZ, M. The transition to adulthood and family relations: an intergenerational perspective. New York: Psychology Press: Taylor \& Francis Group, 2007.

SELLAR, S., GALE, T.; PARKER, S. Appreciating aspirations in Australian higher education. Cambridge Journal of Education, Cambridge, v. 41, n. 1, p. 37-52, Mar. 2011.

SEPÚLVEDA, L.; UGALDE, P. Trayectorias disímiles y proyectos individualizados: origen y experiencia educativo-laboral de los estudiantes de centros de formación técnica. Revista Calidad en la Educación, Santiago, v. 33, p. 63-99, dic. 2010.

SEPÚLVEDA, L.; VALDEBENITO, M. J. ¿Las cosas claras?: aspiraciones de futuro y proyecto educativo-laboral de jóvenes estudiantes secundarios. Estudios Pedagógicos, Valdivia, v. 40, n.1, p. 243-261, 2014.

TOGUCHI, T.; BENGSTON, K. Intergenerational support during the transition to adulthood. In: FURLONG, A. (Ed.) Handbook of youth and young adulthood: new perspectives and agendas. London, Routledge, 2009.

ULE, M.; ŽIVODER, A.; DU BOIS-REYMOND, M. 'Simply the best for my children': patterns of parental involvement in education. International Journal of Qualitative Studies in Education, Indianapolis, v. 28, n. 3, p. 329-348, Feb. 2015.

WARD, S. Neoliberalism and the global restructuring of knowledge and education. London: Routledge, 2012.

WYN, J.; LANTZ, S.; HARRIS, A. Beyond the 'transitions' metaphor: Family relations and young people in late modernity. Journal of Sociology, London, v. 48, n. 1, p. 3-22, Aug. 2011.

\section{NOTAS}

${ }^{1}$ Este artículo se ha desarrollado en el marco del estudio "Modelos de trayectorias y desenlace educativo-laboral de jóvenes: un estudio longitudinal en la Región Metropolitana", Proyecto FONDECYT 1140596. Los enfoques y herramientas metodológicas utilizadas en esta investigación han cumplido con los procedimientos éticos establecidos para la investigación científica de ciencias sociales a nivel nacional.

${ }^{2}$ CONSEJO NACIONAL DE EDUCACIÓN. Índices de Educación Superior. Disponible en: $<$ http://www.cned.cl/>.

${ }^{3}$ OCDE: Organización para la Cooperación y el Desarrollo Económico. 
${ }^{4}$ Durante el año 2015 se inició un proceso, aún incierto, de transición gradual hacia la gratuidad de la educación superior en Chile para los segmentos de menores recursos de la población, bajo un mecanismo de financiamiento estatal. Sus consecuencias son impredecibles, exigiendo un análisis en profundidad de la reorganización institucional asociada y, muy particularmente, de las disposiciones socioculturales del conjunto de la población en la que la asociación esfuerzo económico individual/logro educacional está fuertemente arraigada.

${ }^{5}$ La Encuesta CIDE, que tiene como misión medir bianualmente la opinión de actores del sistema educativo en Chile, constata un incremento sostenido de las expectativas de padres respecto a la finalización de estudios de educación superior de sus hijos. En el año 2000, un $71 \%$ de los encuestados sostenía esta expectativa, mientras que en el año 2012 el porcentaje de casos se había incrementado a un $85 \%$ de los encuestados. Véase $<$ http://educacion. uahurtado.cl/investigacion/encuesta-cide/ $>$.

${ }^{6}$ Fondo Nacional de Desarrollo Científico y Tecnológico de La Comisión Nacional de Investigación Científica y Tecnológica (CONICYT).

${ }^{7}$ Los criterios de selección de la muestra consideraron como unidad primaria de muestreo los establecimientos educativos. Estos fueron seleccionados de manera aleatoria según su dependencia administrativa (pública, privada con financiamiento estatal o privada) y modalidad de estudio. Adicionalmente, en cada una de las unidades, se seleccionó a uno o dos grupos de curso del nivel de $4^{\circ}$ medio (último año de secundaria en el sistema chileno) para la aplicación del instrumento. El cuestionario, que incluyó preguntas destinadas a la caracterización general de los estudiantes, su experiencia educativa, intereses personales y consumo cultural, aspiraciones y proyectos de futuro, entre otras dimensiones, fue de carácter autoaplicado y con la presencia de un encuestador en la sala de clases, quien supervisó el desarrollo del proceso. Las olas siguientes consistieron en encuestas domiciliarias (o lugares de estudio); en los casos en que no fue posible esta modalidad, se aplicó una encuesta telefónica a los jóvenes individualizados.

${ }^{8}$ En el nivel de enseñanza secundaria, el sistema chileno reconoce dos modalidades formativas diferenciadas en los dos últimos años de educación obligatoria, la educación científico-humanista, que prioriza un modelo formativo conducente a estudios superiores entre quienes cursan esta modalidad, y la educación técnico-profesional, que, aunque no inhibe la posibilidad de continuidad de estudios en las distintas alternativas de educación postsecundaria, ofrece una modalidad de formación orientada hacia el mundo del trabajo y el ingreso temprano a una actividad laboral. En esta modalidad concurren estudiantes de los estratos más pobres de la sociedad, representando cerca del $43 \%$ de la matrícula total del nivel correspondiente. Para algunos autores, se trata de un modelo que refuerza las lógicas de segregación social fuertemente presentes en el sistema educacional chileno.

${ }^{9}$ Este criterio es el empleado para organizar la muestra de acuerdo al nivel socioeconómico de las familias de origen y que se reflejan en las tablas contenidas en el presente artículo. La información proviene de los antecedentes aportados por los respectivos jefes de hogar en una encuesta aplicada a nivel nacional a todos los centros educacionales chilenos y que proporciona aclaración sobre ingresos promedios del hogar y nivel educativo alcanzado por padres y/o madres de los estudiantes.

${ }^{10}$ La cuarta etapa de medición en este estudio alcanzó una cobertura de 42,4\% de la muestra inicial, tasa de respuestas que se sitúa en un margen razonable para estudios de estas características. Es lo que se observa (aunque con un tratamiento metodológico y condiciones de realización diferentes), por ejemplo, en los estudios "Socio economic disadvantage and access to higher education" 
realizado en cuatro distritos de Glasgow entre 1999 y 2004; "Paths on life's way", realizado a lo largo de la década de los 90 en British Columbia, Canadá; o el proyecto Life Patterns realizado en Victoria, Australia, en igual período de tiempo. Véase FURLONG, A.; CARTMEL, F., 2005; ANDRES, L.; WYN. J., 2010; BEADLE, S.; HOLDSWORTH, R.; WYN, J., 2011.

${ }^{11}$ Por cierto, este procedimiento de análisis se realizó al conjunto del material generado en el estudio, en el que la dimensión del soporte familiar en una de otras dimensiones que concurren en el análisis de las trayectorias educativo-laborales de los jóvenes estudiantes.

${ }^{12}$ Para el ingreso a una carrera de educación superior universitaria en Chile, los postulantes deben rendir previamente un examen de suficiencia. La prueba de selección universitaria (PSU) es el examen nacional de admisión al sistema universitario chileno. Se realiza una vez al año y, bajo una ruta lógica, los estudiantes rinden esta prueba el último mes del año de egreso de la enseñanza secundaria (aunque, por cierto, existe la alternativa de rendirla en etapas sucesivas posteriormente). El puntaje individual alcanzado es el que permite ulteriormente a un candidato postular a las diversas ofertas existentes en el mercado educativo. El sistema universitario chileno se caracteriza por una amplia heterogeneidad de ofertas, existiendo una separación relevante entre centros de educación superior selectivos (universidades complejas que incluyen mayores exigencias académicas de ingreso a sus postulantes) respectos a centros de educación superior no selectivos (cuyas exigencias académicas de ingreso son menores e incluso, en algunos casos, inexistentes).

${ }^{13}$ Las carreras técnicas de nivel superior son impartidas, en su gran mayoría, por centros privados de educación y, a diferencia de la mayoría del sistema universitario, no requieren la rendición de la prueba nacional de ingreso a la educación superior. Estas carreras pueden ser de 4 u 8 semestres y los costos de ellas, en promedio, son más bajos que los costos de las carreras que se ofrecen en el sistema universitario. En los últimos años ha existido un crecimiento muy relevante de este sistema educativo debido a una mayor participación de estudiantes de estratos bajo y medio- bajo, la proliferación de becas estatales para quienes no pueden financiar sus estudios y la diversificación de la oferta, ampliando alternativas de estudios para quienes desarrollan una actividad laboral.

${ }^{14}$ Por cierto, la experiencia de estudio y trabajo representa una situación cambiante en la trayectoria de los jóvenes de esta investigación; particularmente para quienes no continúan estudios postsecundarios, la actividad laboral se combina con períodos de inactividad a lo largo del período considerado en la indagación.

${ }^{15}$ En esta categoría se incluye el conjunto de respuestas que señalan no tener definido aún un proyecto educativo-laboral o bien aquellas que manifiestan una disposición a destinar un período de tiempo de preparación posescolar para el ingreso a una carrera universitaria. En Chile existe un mercado educativo de preparación para rendir la prueba nacional de ingreso a la educación superior conocido como el mercado de preuniversitarios; aunque muchos jóvenes se incorporen a actividades de este tipo durante su período de formación escolar, existe un grupo relevante de los que lo hacen una vez egresados de la enseñanza secundaria, sobre todo aquellos que han tenido resultados académicos insuficientes en relación a sus intereses vocacionales.

${ }^{16} \mathrm{La}$ categoría otras actividades incluye una proporción muy baja (no superior al 5\% del total) de encuestados que señala no realizar ninguna actividad durante el período; una proporción mayor de jóvenes señala realizar estudios de preparación para rendir el examen de admisión universitario al año siguiente y, particularmente, en los estratos bajo y medio-bajo, existe un segmento de jóvenes que cursaron estudios técnicos en el nivel de enseñanza secundaria, que señala haber realizado su práctica profesional, esto es, un período de trabajo no remunerado en una empresa con el objetivo de obtener el certificado formal de técnico de nivel medio 
que se otorga a quienes cumplen con este requisito curricular. De este modo, es posible señalar que el grueso de lo reportado en la categoría otras actividades corresponde a tareas no permanentes en el tiempo o de tipo transicional en pos de alcanzar un objetivo de corto plazo.

${ }^{17}$ Como puede verse, la presión también declina en el grupo de nivel socioeconómico alto, aunque es posible sostener que las causas son muy distintas al de sus pares más pobres; en este caso, la convicción del cumplimiento de las metas socioeducativas puede ser un factor explicativo de las respuestas particulares de este grupo.

Presentado: 04/01/2016

Aprobado: 26/04/2016

Dirección postal:

Leandro Sepúlveda V.

Facultad de Educación, Universidad Alberto Hurtado

Erasmo Escala 1825 | Santiago de Chile

CEP 8340539 